\title{
Growth and Yield of Sesame, Green Gram and Black Gram as Affected by Different Weed Management Practices
}

\author{
D. Nongmaithem* and D. Pal
}

Dept. of Agronomy, Bidhan Chandra Krishi Viswavidyalaya, Mohanpur, Nadia, West Bengal (741 252), India

\section{Article History}

Manuscript No. AR1644

Received in $25^{\text {th }}$ July, 2016

Received in revised form $30^{\text {th }}$ November, 2016

Accepted in final form $2^{\text {nd }}$ December, 2016

\section{Correspondence to}

*E-mail: debikanong@gmail.com

\section{Keywords}

Black gram, green gram, plant extracts, sesame, weeds

\begin{abstract}
The experiment was conducted at Instructional farm, Jaguli, Bidhan Chandra Krishi Viswavidyalaya, Mohanpur-741252, Nadia,West Bengal during pre-kharif season in 2010 and 2011 to study the growth and yield of sesame, green gram and black gram due to different weed management practices including the botanical plant extracts. The experiment was conducted in split plot design replicated thrice, keeping the crops under the main plot treatment viz. Sesame, Green gram and Black gram and nine weed management treatments in the sub-plot treatments viz. Untreated control, Hand Weeding at $20 \mathrm{DAS}, 5 \%$ (w/v) Ageratum conyzoides aqueous extract, 5\% $(\mathrm{w} / \mathrm{v})$ Blumea lacera aqueous extract, $5 \%(\mathrm{w} / \mathrm{v})$ Ocimum sanctum aqueous extract, $5 \%(\mathrm{w} / \mathrm{v})$ Physalis minima aqueous extract, 5\% (w/v) Amaranthus tricolor aqueous extract, Quizalofop-p-ethyl $50 \mathrm{~g} \mathrm{ha}^{-1}$ at 20 DAS and Fenoxaprop-p-ethyl $30 \mathrm{~g} \mathrm{ha}^{-1}$ at 20 DAS. It was found that black gram and green gram recorded higher control of weeds than sesame. Among the weed management treatments, hand weeding recorded highest weed control efficiency which was followed by fenoxaprop-pethyl. Among the botanical plant extracts, Ageratum conyzoides extract recorded lowest population and dry weight of sedge and broadleaved weeds while Ocimum sanctum extract recorded the same in case of grassy weeds. Hand weeding recorded the highest yield of all the crops. Among the botanical plant extracts Ageratum conyzoides extract recorded highest growth attributes and yield in sesame and black gram while in green gram, Ocimum sanctum extract recorded highest yield.
\end{abstract}

\section{Introduction}

Weeds are the most costly category of agricultural pests, causing great yield loss and labor expense and have been controlled using synthetic herbicides since time immemorial. However, the indiscriminate use of chemical herbicides for weed control has resulted in serious ecological and environmental problems and developed resistance to herbicide in weeds. The increasing concern about the toxicity of synthetic herbicides has boosted the search for eco-friendly and sustainable weed management practices. The management of weeds through natural plant extracts through allelopathy, the phenomenon which is expressed through the release of chemicals by a plant holds great prospect (An et al., 1996) which would diversify weed management practices. Natural compounds from plants provide potential for new herbicidal solutions, or lead compounds for new herbicides (Duke et al., 2000; Vyvyan, 2002) which is due to their likely environmentally benign characteristics (Singh et al., 2003), while the possibility of novel compounds provides the chance of unique modes of herbicidal attack viz., the potential to overcome herbicide resistance.

Allelopathic potential of plant species can be exploited in many ways, and the utilization of the aqueous extracts is one possible tool where the weeds can be better controlled by the utilization of plants that possess a greater fraction of allelochemicals (Elijarrat and Barcelo, 2001). Different plant species contain allelochemicals that vary in type and concentration (Xuan et al., 2005) and the identification of plant species with greater allelopathic potential, and the characterization of their adverse effects against weeds is required for better ecological based weed management.

Several researches conducted on plant extracts for weed control have reported positive views. The control of weeds through the use of plant extracts along with reduction of chemical herbicide doses can also serve as a feasible way of reducing weed growth. Miri and Armin (2013) has found that reducing the rate of herbicide application along with the use of plant extract has resulted in overall yield of wheat. Khan et al. (2015) found that use of several leaf water extract of Eucalyptus, 
Acacia, Sorghum, Shishum, Sunflower, Poplar, Tobacco and Congress grass significantly suppressed weeds by reducing weed density, fresh and dry weed biomass, and encouraged wheat growth and yield.

Therefore, research to discover more natural herbicides from plants needs to be done to find out the potentiality from varying range of plant kingdom having wide abundance not only to reduce the unwanted pollution caused by chemicals but also to reduce the cost of cultivation in crop production.

\section{Materials and Methods}

The field experiment was conducted at the Instructional Farm, Jaguli, BCKV, Mohanpur, Nadia, West Bengal having medium land topography during pre-kharif season of 2010 and 2011. The experimental soil was Gangetic new alluvial (Inceptisol) having good irrigation cum drainage facility with soil $\mathrm{pH}$ of 6.8 , organic carbon- $0.51 \%$, total nitrogen- 250.23 $\mathrm{kg} \mathrm{ha}^{-1}$, available $\mathrm{P}_{2} \mathrm{O}_{5}-21.75 \mathrm{~kg} \mathrm{ha}^{-1}$ and available $\mathrm{K}_{2} \mathrm{O}-181.33$ $\mathrm{kg} \mathrm{ha}^{-1}$. The experiment was conducted in split plot design replicated thrice, keeping the crops (C) under the main plot treatment, $\mathrm{C}_{1}$ : Sesame, $\mathrm{C}_{2}$ : Green gram, $\mathrm{C}_{3}$ : Black gram and nine weed management treatments $(\mathrm{W})$ allocated in the subplot treatments, $\mathrm{W}_{1}$ : Untreated control, $\mathrm{W}_{2}$ : Hand Weeding at 20 DAS, $\mathrm{W}_{3}: 5 \%(\mathrm{w} / \mathrm{v})$ Ageratum conyzoides aqueous extract, $\mathrm{W}_{4}: 5 \%(\mathrm{w} / \mathrm{v})$ Blumea lacera aqueous extract, $\mathrm{W}_{5}: 5 \%(\mathrm{w} / \mathrm{v})$ Ocimum sanctum aqueous extract, $\mathrm{W}_{6}: 5 \%(\mathrm{w} / \mathrm{v})$ Physalis minima aqueous extract, $\mathrm{W}_{7}: 5 \%(\mathrm{w} / \mathrm{v})$ Amaranthus tricolor aqueous extract, $\mathrm{W}_{8}$ : Quizalofop-p-ethyl 5 EC @ $50 \mathrm{~g} \mathrm{ha}^{-1}$ at 20 DAS, $\mathrm{W}_{9}$ : Fenoxaprop-p-ethyl 9 EC @ $30 \mathrm{~g} \mathrm{ha}^{-1}$ at 20 DAS. All the botanical extract treatments were applied as pre-emergence at 1 DAS and added with surfactant Tween $80 @ 0.25 \%$. The varieties of crops used were: Sesame-Rama (Improved Selection-5), Green gram-Bireswar (WBM-34-1-1) and Black gram-Sarada (WBU-108). A spacing of $30 \times 10 \mathrm{~cm}^{2}$ from row to row and plant to plant respectively for all the crops was maintained. The recommended dose of fertilizer for each of the crop in the experiment was applied. Sesame-30:60:30 $\mathrm{kg} \mathrm{ha}^{-1}$ $\mathrm{N}, \mathrm{P}_{2} \mathrm{O}_{5}$ and $\mathrm{K}_{2} \mathrm{O}$ respectively, Green gram and Black gram20:40:40 $\mathrm{kg} \mathrm{ha}^{-1} \mathrm{~N}, \mathrm{P}_{2} \mathrm{O}_{5}$ and $\mathrm{K}_{2} \mathrm{O}$ respectively.

The plant extracts and herbicides were sprayed with a knap sack sprayer with flood jet deflector nozzle size WFN 0.040 with great care to ensure uniform spraying after proper calibration. Aqueous extracts were prepared by following the procedure of Cheema and Khaliq (2000). Leaves of plants were collected from BCKV campus. After collection, leaves were dried in shade at room temperature for a week and later dried at 40 ${ }^{\circ} \mathrm{C}$ in oven for 48 hours and grounded to powder. The dried powder material was soaked in water in the ratio 1:20 (w/v) for 24 hours. Then the water extracts were collected by passing through sieves. The filtrates were boiled at $100{ }^{\circ} \mathrm{C}$ for reducing the volume ( 3 litres). The final extract was left to stand at $4{ }^{\circ} \mathrm{C}$ for 30 minutes and then filtered.

The experimental data were analyzed following the standard statistical method (Panse and Sukhatme, 1985; Gomez and Gomez, 1984).

\section{Results and Discussion}

\subsection{Weed population and dry weight}

At 15 DAS (Table 1), the crops gave no significant effect on all the categories of weeds. This might be attributed to the fact that at this stage the crops did not have the ability of smothering the weeds due to the initial establishment of the crops, as the canopy structure was small enough to hinder the weed growth. Among the weed management treatments, since the botanicals were applied as pre-emergence at 1DAS, there was control of weeds only in botanical plant extract treatments. However, since the hand weeding treatment and chemical herbicide treatments were implemented at 20 DAS, there was no control of the weeds at this stage of observation. At 15 DAS, the population of sedge $\left(3.91 \mathrm{~m}^{-2}\right)$ and broad leaved (4.09 $\mathrm{m}^{-2}$ ) weeds was found to be lowest in Ageratum conyzoides extract. However, lowest population of grass weeds $\left(4.00 \mathrm{~m}^{-2}\right)$ was found in Ocimum sanctum extract which was followed by Ageratum conyzoides extract $\left(4.27 \mathrm{~m}^{-2}\right)$. Idu and Oghale (2013) and Kaur and Sharma (2015) found the potentialilty of Agertaum conyzoides to suppress the growth of weeds. Purohit and Pandya (2013) and Islam and Kato-Noguchi (2014) also reported that aqueous extract of Ocimum species acts as a promising role for the control of weeds since they have phytotoxic substances. The same trend was observed in dry weight of weeds where lowest dry weight of grass weeds $\left(2.73 \mathrm{~g} \mathrm{~m}^{-2}\right)$ was recorded in Ocimum sanctum extract while Ageratum conyzoides extract recorded lowest dry weight of sedge $\left(3.33 \mathrm{~g} \mathrm{~m}^{-2}\right)$ and broad leaved $\left(2.14 \mathrm{~g} \mathrm{~m}^{-2}\right)$ weeds. Among the botanical extract treatments, Amaranthus tricolor extract recorded the highest population of all the weeds but it can be seen from the table that all the botanical extract treatments recorded lower weed population than the control treatment at this stage. The lower population of weeds observed in the botanical plant extract treatments might be due to the presence of suppressive water soluble allelochemicals associated with the respective botanicals. which after application influenced the germination, survival, growth and development of weeds. The inhibition of weeds might have occurred through different toxic mechanism such as reduction in germination or lengthening of germination process or slowing seedling growth which was also reported by Ercoli et al. (2007). Among the botanicals, Amaranthus tricolor extract exhibited the lowest efficiency in controlling of weeds. However, the inhibitory potential of Amaranthus tricolor on some crops was reported by Dhole et 
al. (2013) which might also inhibit weeds.

At 30 and 60 DAS there was significant effect on the population as well as dry weight of the crops. Table 1 and Table 2 showed the weed population and dry weight of weeds respectively where highest population and dry weight of all the categories of weeds was found in sesame while green gram and black gram recorded lower values. This might be attributed to the weed smothering ability of legumes with good canopy were more efficient than sesame for smothering of weeds. Ghosh et al. (2007) also expressed similar opinions, where legumes with good canopy were more efficient than sesame for weed control. Among the weed management treatments, untreated control and hand weeding treatments recorded the highest and lowest population and dry weight respectively of all the categories of weeds. Hand weeding at 20 DAS removed all types of weeds. Hence, at 30 and 60 DAS the lowest population

\begin{tabular}{|c|c|c|c|c|c|c|c|c|c|}
\hline \multirow[t]{2}{*}{ Treatments } & \multicolumn{3}{|c|}{$15 \mathrm{DAS}$} & \multicolumn{3}{|c|}{$30 \mathrm{DAS}$} & \multicolumn{3}{|c|}{$60 \mathrm{DAS}$} \\
\hline & Grass & Sedge & $\begin{array}{c}\text { Broad leaved } \\
\text { weed }\end{array}$ & Grass & Sedge & $\begin{array}{c}\text { Broad leaved } \\
\text { weed }\end{array}$ & Grass & Sedge & $\begin{array}{c}\text { Broad leaved } \\
\text { weed }\end{array}$ \\
\hline \multicolumn{10}{|l|}{ Crops } \\
\hline $\mathrm{C}_{1}$ & $\begin{array}{c}4.85 \\
(23.69)\end{array}$ & $\begin{array}{c}4.69 \\
(21.81)\end{array}$ & $\begin{array}{c}4.56 \\
(20.69)\end{array}$ & $\begin{array}{c}6.28 \\
(41.76)\end{array}$ & $\begin{array}{c}7.76 \\
(61.41)\end{array}$ & $\begin{array}{c}5.18 \\
(27.69)\end{array}$ & $\begin{array}{c}10.20 \\
(104.26)\end{array}$ & $\begin{array}{c}10.86 \\
(118.30)\end{array}$ & $\begin{array}{c}7.66 \\
(59.13)\end{array}$ \\
\hline $\mathrm{C}_{2}$ & $\begin{array}{c}4.62 \\
(21.33)\end{array}$ & $\begin{array}{c}4.75 \\
(22.37)\end{array}$ & $\begin{array}{c}4.71 \\
(21.83)\end{array}$ & $\begin{array}{c}5.91 \\
(36.41)\end{array}$ & $\begin{array}{c}7.30 \\
(54.41)\end{array}$ & $\begin{array}{c}5.02 \\
(25.63)\end{array}$ & $\begin{array}{c}9.96 \\
(100.00)\end{array}$ & $\begin{array}{c}10.43 \\
(109.69)\end{array}$ & $\begin{array}{c}7.07 \\
(50.54)\end{array}$ \\
\hline $\mathrm{C}_{3}$ & $\begin{array}{c}4.43 \\
(19.91)\end{array}$ & $\begin{array}{c}4.65 \\
(21.57)\end{array}$ & $\begin{array}{c}4.59 \\
(20.96)\end{array}$ & $\begin{array}{c}5.26 \\
(29.56)\end{array}$ & $\begin{array}{c}6.42 \\
(43.59)\end{array}$ & $\begin{array}{c}4.65 \\
(22.31)\end{array}$ & $\begin{array}{c}9.70 \\
(94.61)\end{array}$ & $\begin{array}{c}10.25 \\
(105.78)\end{array}$ & $\begin{array}{c}6.41 \\
(41.78)\end{array}$ \\
\hline $\mathrm{SEm} \pm$ & 0.114 & 0.055 & 0.057 & 0.097 & 0.104 & 0.021 & 0.036 & 0.082 & 0.026 \\
\hline $\mathrm{CD}(p=0.05)$ & NS & NS & NS & 0.315 & 0.339 & 0.069 & 0.118 & 0.266 & 0.085 \\
\hline \multicolumn{10}{|c|}{ Weed management treatments } \\
\hline $\mathrm{W}_{1}$ & $\begin{array}{c}5.38 \\
(29.28)\end{array}$ & $\begin{array}{c}5.03 \\
(24.94)\end{array}$ & $\begin{array}{c}4.78 \\
(22.44)\end{array}$ & $\begin{array}{c}7.40 \\
(56.39)\end{array}$ & $\begin{array}{c}8.81 \\
(77.28)\end{array}$ & $\begin{array}{c}5.85 \\
(34.00)\end{array}$ & $\begin{array}{c}11.13 \\
(123.44)\end{array}$ & $\begin{array}{c}11.6 \\
(135.28)\end{array}$ & $\begin{array}{c}8.34 \\
(69.39)\end{array}$ \\
\hline $\mathrm{W}_{2}$ & $\begin{array}{c}4.85 \\
(23.72)\end{array}$ & $\begin{array}{c}4.80 \\
(22.72)\end{array}$ & $\begin{array}{c}4.53 \\
(20.22)\end{array}$ & $\begin{array}{c}3.27 \\
(10.50)\end{array}$ & $\begin{array}{c}4.06 \\
(17.33)\end{array}$ & $\begin{array}{l}2.55 \\
(6.78)\end{array}$ & $\begin{array}{c}8.23 \\
(67.50)\end{array}$ & $\begin{array}{c}8.69 \\
(75.44)\end{array}$ & $\begin{array}{c}5.30 \\
(28.17)\end{array}$ \\
\hline $\mathrm{W}_{3}$ & $\begin{array}{c}4.27 \\
(18.11)\end{array}$ & $\begin{array}{c}3.91 \\
(14.94)\end{array}$ & $\begin{array}{c}4.09 \\
(16.44)\end{array}$ & $\begin{array}{c}6.38 \\
(40.44)\end{array}$ & $\begin{array}{c}6.91 \\
(47.89)\end{array}$ & $\begin{array}{c}5.07 \\
(25.50)\end{array}$ & $\begin{array}{c}10.12 \\
(102.22)\end{array}$ & $\begin{array}{c}10.5 \\
(109.89)\end{array}$ & $\begin{array}{c}6.75 \\
(45.56)\end{array}$ \\
\hline $\mathrm{W}_{4}$ & $\begin{array}{c}4.37 \\
(19.06)\end{array}$ & $\begin{array}{c}4.13 \\
(16.78)\end{array}$ & $\begin{array}{c}4.35 \\
(18.61)\end{array}$ & $\begin{array}{c}6.60 \\
(43.33)\end{array}$ & $\begin{array}{c}7.61 \\
(57.78)\end{array}$ & $\begin{array}{c}5.20 \\
(26.83)\end{array}$ & $\begin{array}{c}10.29 \\
(105.50)\end{array}$ & $\begin{array}{c}10.9 \\
(119.78)\end{array}$ & $\begin{array}{c}7.02 \\
(49.11)\end{array}$ \\
\hline $\mathrm{W}_{5}$ & $\begin{array}{c}4.00 \\
(15.78)\end{array}$ & $\begin{array}{c}4.27 \\
(17.94)\end{array}$ & $\begin{array}{c}4.47 \\
(19.78)\end{array}$ & $\begin{array}{c}6.09 \\
(36.78)\end{array}$ & $\begin{array}{c}7.20 \\
(51.94)\end{array}$ & $\begin{array}{c}5.37 \\
(28.72)\end{array}$ & $\begin{array}{c}9.93 \\
(98.22)\end{array}$ & $\begin{array}{c}10.7 \\
(114.56)\end{array}$ & $\begin{array}{c}7.36 \\
(54.00)\end{array}$ \\
\hline $\mathrm{W}_{6}$ & $\begin{array}{c}4.43 \\
(19.44)\end{array}$ & $\begin{array}{c}4.78 \\
(22.44)\end{array}$ & $\begin{array}{c}4.65 \\
(21.33)\end{array}$ & $\begin{array}{c}6.66 \\
(44.22)\end{array}$ & $\begin{array}{c}7.98 \\
(63.78)\end{array}$ & $\begin{array}{c}5.54 \\
(30.44)\end{array}$ & $\begin{array}{c}10.78 \\
(115.83)\end{array}$ & $\begin{array}{c}11.41 \\
(129.83)\end{array}$ & $\begin{array}{c}7.93 \\
(62.72)\end{array}$ \\
\hline $\mathrm{W}_{7}$ & $\begin{array}{c}4.56 \\
(20.56)\end{array}$ & $\begin{array}{c}4.97 \\
(24.33)\end{array}$ & $\begin{array}{c}4.85 \\
(23.22)\end{array}$ & $\begin{array}{c}7.32 \\
(53.61)\end{array}$ & $\begin{array}{c}8.46 \\
(71.94)\end{array}$ & $\begin{array}{c}5.73 \\
(32.50)\end{array}$ & $\begin{array}{c}11.00 \\
(120.67)\end{array}$ & $\begin{array}{c}11.53 \\
(132.89)\end{array}$ & $\begin{array}{c}8.25 \\
(67.89)\end{array}$ \\
\hline $\mathrm{W}_{8}$ & $\begin{array}{c}4.81 \\
(23.28)\end{array}$ & $\begin{array}{c}5.22 \\
(27.06)\end{array}$ & $\begin{array}{c}4.95 \\
(24.56)\end{array}$ & $\begin{array}{c}4.42 \\
(20.00)\end{array}$ & $\begin{array}{c}6.80 \\
(46.22)\end{array}$ & $\begin{array}{c}4.68 \\
(21.72)\end{array}$ & $\begin{array}{c}9.16 \\
(83.72)\end{array}$ & $\begin{array}{c}9.70 \\
(93.94)\end{array}$ & $\begin{array}{c}6.42 \\
(41.17)\end{array}$ \\
\hline $\mathrm{W}_{9}$ & $\begin{array}{c}5.07 \\
(25.56)\end{array}$ & $\begin{array}{c}5.14 \\
(26.11)\end{array}$ & $\begin{array}{c}4.91 \\
(23.83)\end{array}$ & $\begin{array}{c}4.20 \\
(17.89)\end{array}$ & $\begin{array}{c}6.63 \\
(44.06)\end{array}$ & $\begin{array}{c}4.55 \\
(20.39)\end{array}$ & $\begin{array}{c}8.93 \\
(79.50)\end{array}$ & $\begin{array}{c}9.47 \\
(89.67)\end{array}$ & $\begin{array}{c}6.04 \\
(36.33)\end{array}$ \\
\hline $\mathrm{SEm} \pm$ & 0.124 & 0.064 & 0.069 & 0.135 & 0.136 & 0.100 & 0.080 & 0.083 & 0.094 \\
\hline $\mathrm{CD}(p=0.05)$ & 0.347 & 0.179 & 0.195 & 0.379 & 0.381 & 0.281 & 0.225 & 0.232 & 0.263 \\
\hline
\end{tabular}

$\mathrm{C}_{1}$ : Sesame; $\mathrm{C}_{2}$ : Green gram; $\mathrm{C}_{3}$ : Black gram; $\mathrm{W}_{1}$ : Untreated control; $\mathrm{W}_{2}:$ Hand weeding at $20 \mathrm{DAS} ; \mathrm{W}_{3}: 5 \%(\mathrm{w} / \mathrm{v})$ Ageratum Conyzoides aqueous extract; $\mathrm{W}_{4}: 5 \%(\mathrm{w} / \mathrm{v})$ Blumea lacera aqueous extract; $\mathrm{W}_{5}: 5 \%(\mathrm{w} / \mathrm{v})$ Ocimum sanctum aqueous extract; $\mathrm{W}_{6}: 5 \%(\mathrm{w} / \mathrm{v})$ Physalis minima aqueous extract; $\mathrm{W}_{7}: 5 \%(\mathrm{w} / \mathrm{v})$ Amaranthus tricolor aqueous extract; $\mathrm{W}_{8}$ : Quizalofop-p-ethyl @ $50 \mathrm{~g} \mathrm{a.i} \mathrm{ha}{ }^{-1} ; \mathrm{W}_{9}$ : Fenoxaprop-p-ethyl @ $30 \mathrm{~g}$ a.i. ha $^{-1}$; Figures in the parenthesis are square root transformed values 
Table 2: Effect of treatments on dry weight of weeds $\left(\mathrm{g} \mathrm{m}^{-2}\right)$ at 15 DAS, 30 DAS and 60 DAS (Pooled data)

\begin{tabular}{|c|c|c|c|c|c|c|c|c|c|}
\hline \multirow[t]{2}{*}{ Treatments } & \multicolumn{3}{|c|}{15 DAS } & \multicolumn{3}{|c|}{$30 \mathrm{DAS}$} & \multicolumn{3}{|c|}{$60 \mathrm{DAS}$} \\
\hline & Grass & Sedge & $\begin{array}{c}\text { Broad leaved } \\
\text { weed }\end{array}$ & Grass & Sedge & $\begin{array}{c}\text { Broad } \\
\text { leaved weed }\end{array}$ & Grass & Sedge & $\begin{array}{c}\text { Broad } \\
\text { leaved weed }\end{array}$ \\
\hline \multicolumn{10}{|l|}{ Crops } \\
\hline $\mathrm{C}_{1}$ & $\begin{array}{c}4.85 \\
(23.69)\end{array}$ & $\begin{array}{c}4.69 \\
(21.81)\end{array}$ & $\begin{array}{c}4.56 \\
(20.69)\end{array}$ & $\begin{array}{c}6.28 \\
(41.76)\end{array}$ & $\begin{array}{c}7.76 \\
(61.41)\end{array}$ & $\begin{array}{c}5.18 \\
(27.69)\end{array}$ & $\begin{array}{c}10.20 \\
(104.26)\end{array}$ & $\begin{array}{c}10.86 \\
(118.30)\end{array}$ & $\begin{array}{c}7.66 \\
(59.13)\end{array}$ \\
\hline $\mathrm{C}_{2}$ & $\begin{array}{c}4.62 \\
(21.33)\end{array}$ & $\begin{array}{c}4.75 \\
(22.37)\end{array}$ & $\begin{array}{c}4.71 \\
(21.83)\end{array}$ & $\begin{array}{c}5.91 \\
(36.41)\end{array}$ & $\begin{array}{c}7.30 \\
(54.41)\end{array}$ & $\begin{array}{c}5.02 \\
(25.63)\end{array}$ & $\begin{array}{c}9.96 \\
(100.00)\end{array}$ & $\begin{array}{c}10.43 \\
(109.69)\end{array}$ & $\begin{array}{c}7.07 \\
(50.54)\end{array}$ \\
\hline $\mathrm{C}_{3}$ & $\begin{array}{c}4.43 \\
(19.91)\end{array}$ & $\begin{array}{c}4.65 \\
(21.57)\end{array}$ & $\begin{array}{c}4.59 \\
(20.96)\end{array}$ & $\begin{array}{c}5.26 \\
(29.56)\end{array}$ & $\begin{array}{c}6.42 \\
(43.59)\end{array}$ & $\begin{array}{c}4.65 \\
(22.31)\end{array}$ & $\begin{array}{c}9.70 \\
(94.61)\end{array}$ & $\begin{array}{c}10.25 \\
(105.78)\end{array}$ & $\begin{array}{c}6.41 \\
(41.78)\end{array}$ \\
\hline $\mathrm{SEm} \pm$ & 0.114 & 0.055 & 0.057 & 0.097 & 0.104 & 0.021 & 0.036 & 0.082 & 0.026 \\
\hline $\mathrm{CD}(p=0.05)$ & NS & NS & NS & 0.315 & 0.339 & 0.069 & 0.118 & 0.266 & 0.085 \\
\hline \multicolumn{10}{|c|}{ Weed management treatments } \\
\hline $\mathrm{W}_{1}$ & $\begin{array}{c}5.38 \\
(29.28)\end{array}$ & $\begin{array}{c}5.03 \\
(24.94)\end{array}$ & $\begin{array}{c}4.78 \\
(22.44)\end{array}$ & $\begin{array}{c}7.40 \\
(56.39)\end{array}$ & $\begin{array}{c}8.81 \\
(77.28)\end{array}$ & $\begin{array}{c}5.85 \\
(34.00)\end{array}$ & $\begin{array}{c}11.13 \\
(123.44)\end{array}$ & $\begin{array}{c}11.6 \\
(135.28)\end{array}$ & $\begin{array}{c}8.34 \\
(69.39)\end{array}$ \\
\hline $\mathrm{W}_{2}$ & $\begin{array}{c}4.85 \\
(23.72)\end{array}$ & $\begin{array}{c}4.80 \\
(22.72)\end{array}$ & $\begin{array}{c}4.53 \\
(20.22)\end{array}$ & $\begin{array}{c}3.27 \\
(10.50)\end{array}$ & $\begin{array}{c}4.06 \\
(17.33)\end{array}$ & $\begin{array}{c}2.55 \\
(6.78)\end{array}$ & $\begin{array}{c}8.23 \\
(67.50)\end{array}$ & $\begin{array}{c}8.69 \\
(75.44)\end{array}$ & $\begin{array}{c}5.30 \\
(28.17)\end{array}$ \\
\hline $\mathrm{W}_{3}$ & $\begin{array}{c}4.27 \\
(18.11)\end{array}$ & $\begin{array}{c}3.91 \\
(14.94)\end{array}$ & $\begin{array}{c}4.09 \\
(16.44)\end{array}$ & $\begin{array}{c}6.38 \\
(40.44)\end{array}$ & $\begin{array}{c}6.91 \\
(47.89)\end{array}$ & $\begin{array}{c}5.07 \\
(25.50)\end{array}$ & $\begin{array}{c}10.12 \\
(102.22)\end{array}$ & $\begin{array}{c}10.5 \\
(109.89)\end{array}$ & $\begin{array}{c}6.75 \\
(45.56)\end{array}$ \\
\hline $\mathrm{W}_{4}$ & $\begin{array}{c}4.37 \\
(19.06)\end{array}$ & $\begin{array}{c}4.13 \\
(16.78)\end{array}$ & $\begin{array}{c}4.35 \\
(18.61)\end{array}$ & $\begin{array}{c}6.60 \\
(43.33)\end{array}$ & $\begin{array}{c}7.61 \\
(57.78)\end{array}$ & $\begin{array}{c}5.20 \\
(26.83)\end{array}$ & $\begin{array}{c}10.29 \\
(105.50)\end{array}$ & $\begin{array}{c}10.9 \\
(119.78)\end{array}$ & $\begin{array}{c}7.02 \\
(49.11)\end{array}$ \\
\hline $\mathrm{W}_{5}$ & $\begin{array}{c}4.00 \\
(15.78)\end{array}$ & $\begin{array}{c}4.27 \\
(17.94)\end{array}$ & $\begin{array}{c}4.47 \\
(19.78)\end{array}$ & $\begin{array}{c}6.09 \\
(36.78)\end{array}$ & $\begin{array}{c}7.20 \\
(51.94)\end{array}$ & $\begin{array}{c}5.37 \\
(28.72)\end{array}$ & $\begin{array}{c}9.93 \\
(98.22)\end{array}$ & $\begin{array}{c}10.7 \\
(114.56)\end{array}$ & $\begin{array}{c}7.36 \\
(54.00)\end{array}$ \\
\hline $\mathrm{W}_{6}$ & $\begin{array}{c}4.43 \\
(19.44)\end{array}$ & $\begin{array}{c}4.78 \\
(22.44)\end{array}$ & $\begin{array}{c}4.65 \\
(21.33)\end{array}$ & $\begin{array}{c}6.66 \\
(44.22)\end{array}$ & $\begin{array}{c}7.98 \\
(63.78)\end{array}$ & $\begin{array}{c}5.54 \\
(30.44)\end{array}$ & $\begin{array}{c}10.78 \\
(115.83)\end{array}$ & $\begin{array}{c}11.41 \\
(129.83)\end{array}$ & $\begin{array}{c}7.93 \\
(62.72)\end{array}$ \\
\hline $\mathrm{W}_{7}$ & $\begin{array}{c}4.56 \\
(20.56)\end{array}$ & $\begin{array}{c}4.97 \\
(24.33)\end{array}$ & $\begin{array}{c}4.85 \\
(23.22)\end{array}$ & $\begin{array}{c}7.32 \\
(53.61)\end{array}$ & $\begin{array}{c}8.46 \\
(71.94)\end{array}$ & $\begin{array}{c}5.73 \\
(32.50)\end{array}$ & $\begin{array}{c}11.00 \\
(120.67)\end{array}$ & $\begin{array}{c}11.53 \\
(132.89)\end{array}$ & $\begin{array}{c}8.25 \\
(67.89)\end{array}$ \\
\hline $\mathrm{W}_{8}$ & $\begin{array}{c}4.81 \\
(23.28)\end{array}$ & $\begin{array}{c}5.22 \\
(27.06)\end{array}$ & $\begin{array}{c}4.95 \\
(24.56)\end{array}$ & $\begin{array}{c}4.42 \\
(20.00)\end{array}$ & $\begin{array}{c}6.80 \\
(46.22)\end{array}$ & $\begin{array}{c}4.68 \\
(21.72)\end{array}$ & $\begin{array}{c}9.16 \\
(83.72)\end{array}$ & $\begin{array}{c}9.70 \\
(93.94)\end{array}$ & $\begin{array}{c}6.42 \\
(41.17)\end{array}$ \\
\hline $\mathrm{W}_{9}$ & $\begin{array}{c}5.07 \\
(25.56)\end{array}$ & $\begin{array}{c}5.14 \\
(26.11)\end{array}$ & $\begin{array}{c}4.91 \\
(23.83)\end{array}$ & $\begin{array}{c}4.20 \\
(17.89)\end{array}$ & $\begin{array}{c}6.63 \\
(44.06)\end{array}$ & $\begin{array}{c}4.55 \\
(20.39)\end{array}$ & $\begin{array}{c}8.93 \\
(79.50)\end{array}$ & $\begin{array}{c}9.47 \\
(89.67)\end{array}$ & $\begin{array}{c}6.04 \\
(36.33)\end{array}$ \\
\hline $\mathrm{SEm} \pm$ & 0.124 & 0.064 & 0.069 & 0.135 & 0.136 & 0.100 & 0.080 & 0.083 & 0.094 \\
\hline $\mathrm{CD}(p=0.05)$ & 0.347 & 0.179 & 0.195 & 0.379 & 0.381 & 0.281 & 0.225 & 0.232 & 0.263 \\
\hline
\end{tabular}

Figures in the parenthesis are square root transformed values

as well as dry weight of all the categories of weeds was recorded in hand weeding treatment. Tamang et al. (2015) also reported similar findings in green gram. The same treatment recorded the highest weed control efficiency of $69.73 \%$ at 30 DAS (Table 3). After the application of chemical herbicides at 20 DAS, there was reduction in the population of weeds at 30 and 60 DAS, where the reduction was more pronounced in grass weed. Mehmood et al. (2014) also obtained similar type of results where application of fenoxaprop-ethyl resulted in a significant reduction in monocotyledonous weeds. Among the two herbicides used in the experiment, Fenoxaprop-p-ethyl exhibited more suppression of all categories of weeds than quizalofop-p-ethyl $\left(\mathrm{W}_{8}\right)$. It can be seen from Table 1 that at 30 and 60 das, the population of weeds under fenoxaprop-pethyl treatment is significantly lower than quizalopfop-p-ethyl treatment which might be due to the contact as well as systemic action of fenoxaprop-p-ethyl. Similar type of observations was also reported by Sitangshu (2006).

Among the botanical plant extracts, the trend observed at 15 DAS was same as at 30 and 60 DAS. However, the control of weeds by the botanicals decreased with respect to 15 DAS. The probable reason might be due to the degradation of chemicals 
present in the extracts which might be in the form of leaching or volatilization. Bailey et al. (2010) also reported the loss of botanical chemical through leaching. Hence, the suppressing ability of the allelochemicals reduced leading to the regaining of growth of weeds and ultimately increased the dry weights of all the weeds. The degree of suppression of weeds by different botanical treatments vary among themselves and this might be due to the concentration dependent factor of the allelopathic plant species as also stated by Ismail and Chong (2007).

\subsection{Growth attributes and seed yield}

The growth attributes in different crops differed significantly and it was partly due to the weed management treatments and largely due to their genetic inherent characteristics.

The hand weeding treatment recorded the highest dry matter accumulation (Table 3$)$ in all the crops $\left(360.54 \mathrm{~g} \mathrm{~m}^{-2}\right.$ in sesame, $212.58 \mathrm{~g} \mathrm{~m}^{-2}$ in green gram and $293.06 \mathrm{~g} \mathrm{~m}^{-2}$ in black gram) followed by fenoxaprop-p-ethyl treatment. The same trend was also observed in crop growth rate where the highest was found in hand weeding treatment $\left(6.84 \mathrm{~g} \mathrm{~m}^{-2} \mathrm{~d}^{-1}\right)$ in sesame, $\left(4.29 \mathrm{~g} \mathrm{~m}^{-2} \mathrm{~d}^{-1}\right)$ in green gram and $\left(4.17 \mathrm{~g} \mathrm{~m}^{-2} \mathrm{~d}^{-1}\right)$ in black gram. In the case of seed yield of crops, it was also found that the highest seed yield in sesame, green gram and black gram

Table 3: Effect of treatments on dry matter accumulation, crop growth rate (30-45 DAS) and weed control efficiency at 30 DAS (Pooled data)

\begin{tabular}{|c|c|c|c|c|c|c|c|c|c|c|c|c|}
\hline \multirow{3}{*}{$\begin{array}{l}\text { Weed manage- } \\
\text { ment treatments }\end{array}$} & \multicolumn{12}{|c|}{ Crops } \\
\hline & \multicolumn{4}{|c|}{ Dry matter accumulation $\mathrm{g} \mathrm{m}^{-2}$} & \multicolumn{4}{|c|}{ Crop growth rate $\mathrm{g} \mathrm{m}^{-2} \mathrm{~d}^{-1}$} & \multicolumn{4}{|c|}{ Weed control efficiency \% } \\
\hline & $\mathrm{C}_{1}$ & $\mathrm{C}_{2}$ & $\mathrm{C}_{3}$ & Mean & $\mathrm{C}_{1}$ & $\mathrm{C}_{2}$ & $\mathrm{C}_{3}$ & Mean & $\mathrm{C}_{1}$ & $\mathrm{C}_{2}$ & $\mathrm{C}_{3}$ & Mean \\
\hline $\mathrm{W}_{1}$ & 194.44 & 150.58 & 170.18 & 171.73 & 3.23 & 2.62 & 2.22 & 2.69 & - & - & - & - \\
\hline $\mathrm{W}_{2}$ & 360.54 & 212.58 & 293.06 & 288.73 & 6.84 & 4.29 & 4.17 & 5.10 & 64.90 & 70.35 & 73.95 & 69.73 \\
\hline $\mathrm{W}_{3}$ & 248.64 & 172.47 & 227.39 & 216.17 & 3.70 & 3.32 & 3.33 & 3.45 & 28.53 & 31.04 & 38.20 & 32.59 \\
\hline $\mathrm{W}_{4}$ & 231.96 & 159.37 & 193.74 & 195.02 & 3.64 & 3.03 & 2.67 & 3.11 & 19.96 & 22.38 & 27.51 & 23.29 \\
\hline $\mathrm{W}_{5}$ & 241.94 & 181.68 & 222.67 & 215.43 & 3.59 & 3.54 & 3.18 & 3.44 & 25.66 & 26.57 & 33.77 & 28.67 \\
\hline $\mathrm{W}_{6}$ & 234.69 & 164.97 & 212.62 & 204.10 & 3.74 & 3.16 & 2.90 & 3.27 & 13.20 & 14.66 & 17.57 & 15.14 \\
\hline $\mathrm{W}_{7}$ & 206.95 & 154.74 & 201.90 & 187.86 & 3.26 & 2.94 & 2.92 & 3.04 & 8.59 & 10.67 & 9.07 & 9.44 \\
\hline $\mathrm{W}_{8}$ & 283.21 & 198.55 & 276.43 & 252.73 & 4.55 & 3.99 & 3.99 & 4.18 & 42.19 & 55.62 & 58.61 & 52.14 \\
\hline $\mathrm{W}_{9}$ & 292.82 & 202.51 & 280.58 & 258.64 & 4.55 & 4.11 & 4.10 & 4.26 & 46.45 & 59.91 & 67.26 & 57.87 \\
\hline Mean & 255.02 & 177.49 & 230.95 & & 4.12 & 3.45 & 3.28 & & 27.72 & 32.36 & 36.22 & \\
\hline Interaction & $\mathrm{C}$ & W & $\mathrm{C} \times \mathrm{W}$ & $\mathrm{W} \times \mathrm{C}$ & $\mathrm{C}$ & W & $\mathrm{C} \times \mathrm{W}$ & $\mathrm{W} \times \mathrm{C}$ & & & & \\
\hline $\mathrm{SEm} \pm$ & 1.690 & 2.613 & 4.526 & 4.590 & 0.066 & 0.127 & 0.219 & 0.217 & & & & \\
\hline $\mathrm{CD}(p=0.05)$ & 5.512 & 7.336 & 12.870 & 14.886 & 0.216 & 0.356 & 0.624 & 0.692 & & & & \\
\hline
\end{tabular}

C: Crops; W: Weed management

was found in hand weeding treatment $\left(0.91 \mathrm{tha}^{-1}, 1.36 \mathrm{tha}^{-1}\right.$ and $1.67 \mathrm{t} \mathrm{ha}^{-1}$ respectively) followed by fenoxaprop-p-ethly treatment for all the crops (Table 4). Chaudhari et al. (2016) also reported from their research that hand weeding gave the best result in green gram. The lowest seed yield of the crops was recorded in weedy check. $0.61 \mathrm{tha}^{-1}$ in sesame, $0.93 \mathrm{tha}^{-1}$ in green gram and $1.12 \mathrm{t} \mathrm{ha}^{-1}$ in black gram. Highest weed competition in untreated control hindered crop growth resulting lowest values of yield in all the crops. In the case of botanical extract treatments, Ageratum conyzoides extract recorded the highest dry matter accumulation in sesame $\left(248.64 \mathrm{~g} \mathrm{~m}^{-2}\right)$ and black gram $\left(227.39 \mathrm{~g} \mathrm{~m}^{-2}\right)$ followed by Ocimum sanctum extract in both the crops $\left(241.94 \mathrm{~g} \mathrm{~m}^{-2}\right.$ in sesame and 222.67 $\mathrm{g} \mathrm{m}^{-2}$ in black gram while Ocimum santum extract recorded the highest dry matter accumulation in green gram $(181.68 \mathrm{~g}$ $\mathrm{m}^{-2}$ ) followed by Ageratum conyzoides extract $\left(172.47 \mathrm{~g} \mathrm{~m}^{-2}\right)$.
The trend was same for crop growth rate and seed yield of all the crops. It may be noted that Ageratum conyzoides extract in spite of recording highest weed control among the botanicals, it was unable to obtain the best result in growth attributes and yield in green gram. The possible reason might be due to some inhibitory action of the extract of Ageratum conyzoides on green gram crop. Bhatt et al. (2001) working with different plant extracts including Ageratum conyzoides on green gram in laboratory condition found that the aqueous extracts of dried fresh leaves of the weed significantly suppressed the germination, plumule and radical of the crop. However, in the results obtained from the experiment, there was no such drastic effect on the crop. The probable reason might be due to low concentration of the extract used or due to some external environmental factors prevailing in the field condition which neutralize the suppressing action of allelochemicals. 


\begin{tabular}{|c|c|c|c|c|c|c|c|c|}
\hline \multirow{3}{*}{$\begin{array}{l}\text { Weed manage- } \\
\text { ment treatments }\end{array}$} & \multicolumn{8}{|c|}{ Crops } \\
\hline & \multicolumn{4}{|c|}{ Seed yield $t \mathrm{ha}^{-1}$} & \multicolumn{4}{|c|}{ Weed index \% } \\
\hline & $\mathrm{C}_{1}$ & $\mathrm{C}_{2}$ & $\mathrm{C}_{3}$ & Mean & $\mathrm{C}_{1}$ & $\mathrm{C}_{2}$ & $\mathrm{C}_{3}$ & Mean \\
\hline $\mathrm{W}_{1}$ & 0.61 & 0.93 & 1.12 & 0.89 & 32.27 & 30.93 & 32.86 & 32.02 \\
\hline $\mathrm{W}_{2}$ & 0.91 & 1.36 & 1.67 & 1.31 & 0.00 & 0.00 & 0.00 & 0.00 \\
\hline $\mathrm{W}_{3}$ & 0.77 & 1.15 & 1.37 & 1.10 & 14.77 & 14.29 & 17.86 & 15.64 \\
\hline $\mathrm{W}_{4}$ & 0.71 & 1.05 & 1.19 & 0.98 & 20.94 & 22.01 & 28.91 & 23.95 \\
\hline $\mathrm{W}_{5}$ & 0.73 & 1.20 & 1.34 & 1.09 & 18.59 & 11.04 & 19.54 & 16.39 \\
\hline $\mathrm{W}_{6}$ & 0.69 & 1.10 & 1.28 & 1.02 & 23.96 & 18.10 & 23.53 & 21.86 \\
\hline $\mathrm{W}_{7}$ & 0.66 & 0.99 & 1.22 & 0.96 & 27.34 & 26.73 & 26.81 & 26.96 \\
\hline $\mathrm{W}_{8}$ & 0.83 & 1.30 & 1.48 & 1.20 & 8.41 & 3.22 & 11.75 & 7.79 \\
\hline $\mathrm{W}_{9}$ & 0.85 & 1.33 & 1.49 & 1.22 & 5.72 & 1.34 & 10.68 & 5.91 \\
\hline Mean & 0.75 & 1.16 & 1.35 & & 16.89 & 14.18 & 19.10 & \\
\hline Interaction & $\mathrm{C}$ & W & $\mathrm{C} \times \mathrm{W}$ & $\mathrm{W} \times \mathrm{C}$ & & & & \\
\hline $\mathrm{SEm} \pm$ & 0.010 & 0.016 & 0.027 & 0.027 & & & & \\
\hline $\mathrm{CD}(p=0.05)$ & 0.031 & 0.044 & 0.077 & 0.089 & & & & \\
\hline
\end{tabular}

The high seed yield in hand weeding at $20 \mathrm{DAS}$, might be due to the removal of weeds which coincide with the critical period of crop weed competition of sesame (15-45 DAS) which was also reported by Duary and Hazra (2013) and 20-40 DAS in case of green gram and black gram which was also opined by Sheoran et al. (2008). The weed management treatments recorded higher seed yield over untreated control and it may be due to direct or indirect expression of a reduction in weedcrop competition which helped to increased seed yield. Singh et al. (2001) also reported similarly.

\subsection{Weed control efficiency and weed index}

At 30 DAS, highest weed control efficiency of $64.90 \%$, $70.35 \%$ and $73.95 \%$ was recorded by hand weeding treatment in sesame, green gram and black gram respectively which was followed by fenoxaprop-p-ethyl, which is due to better control of weeds and reduction of crop weed competition throughout the growth stages of the crop. Among the botanical plant extracts, Ageratum conyzoides extract recorded the highest weed control efficiency in all the crops $(28.53 \%$ in sesame, $31.04 \%$ in green gram and $38.20 \%$ in black gram) which was followed by Ocimum sanctum extract. In the case of weed index the highest is recorded in untreated control recording $32.27 \%$ in sesame, $30.93 \%$ in green gram and $32.86 \%$ in black gram. Mansoori et al. (2015) reported that unchecked weeds caused a reduction of $66.67 \%$ in black gram yield. The lowest weed index in sesame (14.77\%) and black gram (17.86\%) was recorded in Ageratum conyzoides extract while it found to be the lowest in Ocimum sanctum extract in green gram (11.04\%).

\section{Conclusion}

Use of botanical plant extracts for weed management can change the dynamics of weed population where Ageratum conyzoides extract@5\% (w/v) can be used as pre-emergence herbicide for controlling weeds particularly sedge and broadleaved weeds and Ocimum sanctum extract@5\% (w/v) for grassy weeds in the initial stage of crop growth.

\section{References}

An, M., Pratley, J.E., Haig, T., 1996. Allelopathy: from concept to reality. Proceedings of the $8^{\text {th }}$ Australian Agronomy Conference, Toowoomba, 616.

Bailey, K.L., Pitt, W.M., Derby, J., Walter, S., Taylor, W., Falk, S., 2010. Efficacy of Phoma macrostoma, a Bioherbicde, for control of dandelion (Taraxacum officinale) following simulated rainfal condition. The Americas Journal of Plant Science and Biotechnology 4(2), 35-42.

Bhatt, B.P., Tomar, J.M.S., Misra, L.K., 2001. Allelopathic effects of weeds on germination and growth of legumes and cereal crops of North Eastern Himalayas. Allelopathy Journal 8(2), 225-232.

Chaudhari, V.D., Desai, L.J., Chaudhari, S.N., Chaudhari, P.R., 2016. Effect of weed management on weeds, growth and yield of summer green gram (Vigna radiata L.). The Bioscan 11(1), 531-534.

Chauhan, D.V.S., Gurjar, B.S., 1998. Mechanical and chemical weed control in sesame (Sesamum indicum). Indian Journal of Agronomy 43(3), 480-483.

Cheema, Z.A., Khaliq, A., 2000. Use of sorghum allelopathic 
properties to control weeds in irrigated wheat in a semi-arid region of Punjab. Agriculture, Ecosystem and Environment 79, 105-112.

Duary, B., Hazra, D., 2013. Determination of critical period of crop-weed competition in sesame. Indian Journal of Weed Science 45(4), 253-256.

Dhole, J.A., Lone, K.D., Dhole, G.A., Bodke, S.S., 2013. Allelopathic effect of aqueous and ethanolic extracts of some common weeds on seed health of Triticum aestivum L. (Wheat). International Journal of Current Microbiology and Applied Sciences 2(6), 254-260.

Duke, S.O., Romagni, J.G., Dayan, F.E., 2000. Natural products as sources for new mechanisms of herbicidal action. Crop Protection 19, 583-589.

Elijarrat, E., Barcelo, D., 2001. Sample handling and analysis of allelochemical compounds in plants. Trends in Analytical Chemistry 20(10), 584-590.

Ercoli, L., Masoni, A., Pampana, S., Arduini, I., 2007. Allelopathic effects of rye, brown mustard and hairy vetch on redroot pigweed, common lambsquarter and knotweed. Allelopathy Journal 19(1), 249-256.

Ghosh, P.K., Bandyopadhyay, K.K., Wanjari, R.H., Manna, M.C., Misra, A., Mohanty, K., Subba Rao, M.A., 2007. Legume effect for enhancing productivity and nutrient use-efficiency in major cropping systems-an indian perspective: A Review Journal of Sustainable Agriculture 30(1), 59-86.

Gomez, K.A., Gomez, A.A., 1984. Statistical procedures for agricultural research. Second Edition. John Wiley and Sons, New York, USA.

Idu, M.D., Oghale, O.V., 2013. Studies on the allelopathic effect of aqueous extract of Ageratum conyzoides asteraceae on seedling growth of Sesanum indicum L. (pedaliaceae). International Journal of Science, Environment and Technology 2(6), 1185-1195.

Islam, A.K.M.M., Kato-Noguchi, H., 2014. Phytotoxic activity of Ocimum tenuiflorum extracts on germination and seedling growth of different plant species. Available from: http: /dx. doi. org/10. 1155/2014/676242. $10.1155 / 2014 / 676242$

Ismail, B.S., Chong, T.V., 2007. Effects of the extract of Dicranopteris linearis on seed germination and seedling growth of selected common weeds in Malaysia. Allelopathy Journal 20(2), 287-296.

Kaur, I., Sharma, R., 2015. Allelopathic effect of Ageratum conyzoides L. on protein content of the mungbean (Vigna radiata L.) plants. Indian Journal of Applied and Pure
Biology 30(1), 7-10.

Khan, E.A., Khakwani, A.A., Munir, M., Ghazanfarullah., 2015. Effects of allelopathic chemicals extracted from various plant leaves on weed control and wheat crop productivity. Pakistan Journal of Botany 47(2), 735-740.

Khan, R., Khan, M.A., 2012. Weed control efficiency of bioherbicides and their impact on grain yield of wheat (Triticum aestivum L.). European Journal of Applied Sciences 4(5), 216-219.

Mansoori, N., Bhadauria, N., Rajput, R.L., 2015. Effect of weed control practices on weeds and yield of black gram (Vigna mungo). Legume Research 38(6), 855-857.

Miri, H.R., Armin, M., 2013. The use of plant water extracts in order to reduce herbicide application in wheat. European Journal of Experimental Biology 3(5),155-164.

Panse, V.G., Sukhatme, P.V., 1985. Statistical method of Agricultural workers. ICAR, New Delhi, 152-159.

Purohit, S., Pandya, N., 2013. Allelopathic activity of Ocimum sanctum L. and Tephrosia purpurea (L.) Pers. leaf extracts on few common legumes and weeds. International Journal of Research in Plant Science 3(1), 5-9.

Sheoran, P., Singh, S., Sardana, V., Bawa, S.S., 2008. Studies on critical period of criop weed competition in green gram in Kandi region of Punjab. Indian Journal of Dryland Agricultural Research and Development 23(1), 19-22.

Singh, H.P., Batish, D.R., Kohli, R.K., 2003. Allelopathic interactions and allelochemicals: new possibilities for sustainable weed management. Critical Reviews in Plant Sciences 22, 239-311.

Singh, H., Singh, B.P., Prasad, H., 2001. Weed management in Brassica species. Indian Journal of Agronomy 46(3), 533-537.

Sitangshu, S., 2006. Weed management in jute (Corchorus olitorius L.) by post emergence herbicides. Journal of Tropical Agriculture 44(1/2), 71-73.

Tamang, D., Nath, R., Sengupta, K., 2015. Effect of herbicide application on weed management in green gram [Vigna radiata (L.) Wilczek]. Advances in Crop Science and Technology 3(2), 163.

Vyvyan, J.R., 2002. Allelochemicals as leads for new herbicides and agro-chemicals. Tetrahedron 58, 1631-1636.

Xuan, T.D., Hong, N.H., Khanh, T.D., Eiji, T., Tawata, S., Fukuta, M., 2005. Biological control of weeds and plant pathogens in paddy fields by exploiting plant allelopathy: an overview. Crop Protection, 24(1), 197-206. 